\title{
Social networks and psychological safety: A model of contagion
}

\author{
André Escórcio Soares ${ }^{1}$, Miguel Pereira Lopes ${ }^{2}$ \\ ${ }^{1}$ Polytechnic Institute of Tomar (Portugal) \\ ${ }^{2}$ School of Social and Political Sciences, University of Lisbon and CAPP - \\ Public Policies and Administration Center (Portugal) \\ andre.escorcio@ipt.pt.mplopes@iscsp.ulisboa.pt
}

Received: March 2014

Accepted: August 2014

\section{Abstract:}

Purpose: We attempted to explain how the interactions between members influence the psychological safety of a team using social network analysis by proposing a model based on social contagion in which the psychological safety of the central member has a key role in the psychological safety of the whole team.

Design/methodology: We present a theoretical paper which crosses theory about social network analysis, psychological safety and social contagion.

Findings: We suggest that there are two groups of variables that mediate this relationship. The first group concerns the characteristics of the node and is composed by the proximity to the node's personal characteristics and the value of the central member as a source of information. Second, we advance that there are two dimensions at the level of tie properties that mediate the influence of a central member on team psychological safety - tie strength and friendship level. Finally, the interacting opportunities- a variable at context level - is considered to affect the strength of the ties. We also advance some variables that mediate the influence of the psychological safety of a central member on the psychological safety of the team. 
Originality/value: To the best of our knowledge there is no significant research using social network analysis to explain the process by which a team becomes psychologically safe. On the other hand, because psychological safety tends to be a team construct it is important to understand how team dynamics, evidenced by social network analysis, influence the formation of psychological safety through contagion processes.

Keywords: Psychological safety, Teams, Social network analysis, Contagion

\section{Introduction}

In the last years several studies have tried to shed light on what makes a "good team". However, they focused on an input-process-output framework, i.e. they have generally tried to understand the properties (e.g. size, ability, etc.) that produce desirable behaviours (e.g. quality of group performance), emotions (e.g. satisfaction) and other results (see Gist, Locke, \& Taylor, 1987 for a review). Contrarily, Stacey (1996) states that teams are non-linear feedback networks that are continuously involved in ongoing processes of positive and negative feedback. On the other hand, Kozlowski and Ilgen (2006, pp. 78) argue that "teams are complex dynamic systems that exist in a context, develop as members, interact over time, and evolve and adapt as situational demands unfold". In this sense it is important to understand how the interaction between team members affects team functioning. So the traditional linear perspective has failed to capture the complex dynamics inherent in these strong interaction processes (Losada \& Heaphy, 2004). In line with these perspectives, to have a clear understanding of the functioning of teams and to predict their effectiveness it is crucial to study personal relations within teams. In this sense, social network analysis (SNA) is a useful methodology to understand the intra-group relations, specially the informal relations within a team and consequently their result. For example, Coleman (1988) stressed the positive effect of cohesive social ties on the existence of effective norms and on the trustworthiness of social structures which diminishes the uncertainty of their exchanges and enhances their ability to cooperate.

On the other hand, the social environment in which these interactions occur plays a key role on team performance and effectiveness. In this article we intend to explore a specific dimension of the social environment - the psychological safety - defined as a shared belief held by the members of a team that the team is safe for interpersonal risk-taking (Edmondson, 1999). Everyone has experienced situations in which they felt that the social environment was not safe to take interpersonal risk and, because of that, avoided to interact with others. For example, when a student notices a lecturer made a mistake he may feel reluctant in pointing out the mistake. This happens, not only because he is afraid of the lecturer's reaction, but also because he is afraid of the reaction of the entire class. This also happens in organizations, for 
example when a worker finds an error on the system but doesn't feel comfortable to talk about it. Edmondson (1999) advanced that individuals act like this in order to protect their personal image. For example, if a worker admits the error he may be seen as a negative person.

In this sense psychological safety is an individual psychological state (rather than a personal trait) in which individuals feel that the social context is not threatening, and it is safe for them to express themselves without hurtful consequences. According to Baer and Frese (2003), an individual's psychological safety reflects a work environment where employees can speak out without negative consequences. They refer to this safe environment as organizational climate for psychological safety. However psychological safety tends to be studied more at an individual or team level. For example, Edmondson (1999) related team psychological safety with learning behaviours. This author refers to psychological safety as a team concept. In this sense, the individual evaluation of the interpersonal risk should converge in the entire team. The research about team psychological safety focuses mainly on its antecedents and consequences. May, Gilson and Harter (2004), for example, focused on the influence of coworker relations, supervisor relations and co-worker norms as antecedents of team psychological safety. On the other hand, Carmeli (2007) advanced that psychological safety is positively associated with failure-based learning behaviours. In the present paper, we advance a model focused on the team dynamics, i.e. the communication network, to explain the emergence of the psychological safety. Based on several studies (e.g., Edmondson, 1999; Edmondson, 2003; May et al., 2004; Baer \& Frese, 2003) we assume that higher levels of psychological safety will positively influence team and firm performance as well as team learning behaviours.

To the best of our knowledge there is no significant research using social network analysis to explain the process by which a team becomes psychologically safe. On the other hand, because psychological safety tends to be a team construct it is important to understand how team dynamics, evidenced by social network analysis, influence the formation of psychological safety through contagion processes. The goal of this paper is thus to propose a model to understand the influence of the features of the team social network on the psychological safety of team members through a social contagion process. In addition, we expect to contribute to the progress of the psychological safety field proposing a model to explain the emergence of psychological safety through the interactions between team members. Finally, we intend to make a call for further theoretical research to explain the process by which a team becomes psychologically safe.

In the remainder of the paper, we begin by reviewing the literature about psychological safety and social network analysis. Then we present an analysis of the interaction between team social network and psychological safety, and present the theoretical model. We conclude by discussing the implications of our theoretical propositions. 


\section{Psychological Safety}

According to Edmondson (1999), psychological safety is defined as a shared belief that the team is safe for interpersonal risk-taking. Individuals within teams tend to adopt passive behaviours concerning their personal relationships, in order to protect their personal image.

Edmondson (2003) states four personal risks that individuals face when working in teams:

- when someone makes a question may be seen as ignorant;

- when someone admits an error (or simply calls attention to it), asks for help or accepts the probability of failing, risks being seen as incompetent in general or in that specific task;

- if someone who criticizes past or present events, may be catalogued as negative;

- finally to avoid being seen as intrusive, people tend not to ask for feedback.

The psychological safety of a team is distinct from other relational constructs such as trust and perceived organizational support (Carmeli \& Gifttell, 2009). Psychological safety goes beyond interpersonal trust as it refers to the climate within a team characterized by both interpersonal trust and mutual respect that allows people to be themselves (Edmondson, 1999). However Edmondson (1999) admits that trust may provide a foundation for further development of the interpersonal beliefs that constitute the psychological safety of a team.

Carmeli and Gifttell (2009) stressed that perceived organizational support is a related concept but not necessarily the same thing. Perceived organizational support emphasises the general beliefs about the appreciation of the organization for the work of their employees and the concern with their well-being (organization focused), while psychological safety is about feeling comfortable to take interpersonal risks (personal relations focused).

Because psychological safety is a belief, it should converge in a team once the team members are subject to the same set of structural influences and these perceptions develop out of salient experiences (Edmondson, 1999). This is consistent with the social processing theory of Salancik and Pfeffer (1978) which advocates that the social environment provides cues which individuals use to make sense about reality and also provides information about what attitudes and behaviours are expected by the group. For example, if a team member is punished every time he/she makes a mistake, the team members feel that they cannot admit a mistake or will be punished. In this sense, the shared experience of team members ("a team member is punished every time he/she makes a mistake") is fundamental for the development of a common belief ("it is wrong to admit a mistake") and influences the individual behaviour ("don't admit mistakes"). In this case, the low psychological safety has been generated by the social context and previous experience. 
According to May et al. (2004), the determinants of psychological safety include supportive supervisory relations, rewarding co-worker relations and adherence to behavioural norms. First, the relationship with one's supervisor can have an impact on an individual's perceptions of the safety of a work environment. Edmondson (1999) pointed that a supportive and coaching oriented leader that has non-defensive responses to questions and challenges will enable the psychological safety of the team members. Second, May et al. (2004) found that co-workers who support each other during tough times at work have mutual respect for one another and value each other's contributions help increase the levels of psychological safety. Third, May et al. (2004) found that normative rules in teams lead to feelings of low psychological safety. They refer to these normative rules as implicit norms that team members follow. So when team members tend to converge to shared normative values, they tend to act according to those rules and to avoid questioning the customary behaviour routines. In this sense, the team members will not take any interpersonal risk, which means they will have low psychological safety. Psychological safety has also been studied mainly as a predictor of learning behaviours (e.g. Edmondson, 1999; Carmeli \& Gittell, 2009), firm performance (e.g. Baer \& Frese, 2003), work engagement (e.g. Mayet al., 2004) and accident prevention (Probst \& Estrada, 2010). Despite psychological safety being related to interpersonal risk-taking, the direct influence of social networks on the psychological safety of a team has received little attention. Due to this, the main goal of this paper is to propose a model to understand the influence of the social network features of a team on the psychological safety of its members.

\section{Social Network Analysis}

Social network research has attracted attention from different fields such as sociology, economics, anthropology, mathematics, political science, history and social psychology (Lusher, Robins \& Kremer, 2010). Borgatti and Foster (2003) pointed out that the network literature has been growing exponentially since the second half of the 20th century, part of a general shift away from individualist, essentialist and atomistic explanations toward a more relational, contextual and systemic understanding. An advantage of social network analysis is the ability to analyse both the individual attributes (e.g. preferences, skills, abilities, etc.) and social structures (e.g. information flow within a team) (Robins \& Kremer, 2010). In this sense, social network analysis might be a powerful tool to investigate complex relations both in organizations and teams.

There are many concepts related to social network analysis. However, for the purpose of this paper, only the more important ones are referred to. A key concept in social network analysis has been the notion of centrality. This refers to the importance of a node according to its structural position in the network. There are several concepts about centrality. One of the most known concepts related to centrality is closeness, a concept advanced by Freeman (1978) who defined it as the sum of the distances to or from all other nodes, where distance is defined 
graph-theoretically in terms of the number of links in the shortest path between two nodes. Closeness is usually measured by averaging the path distances (direct and indirect links) to all the others. In this sense, direct links are counted as one and indirect links receive proportionately less weight.

We have a structural hole when two non-connected actors are connected to the same actor. The number of structural holes allows us to estimate the sparseness or closure of an ego network, and may be considered as a density measure when we consider the total number of structural holes in the whole network. According to Burt (2005, pp.16), "structural holes are the empty spaces in social structure".

On the other hand, closure is the opposite of a structural hole and is measured as ego-network density. A network with complete closure is one in which all actors are connected to one another (see for example Figure 1). In such cases, density reaches its theoretical maximum (Coleman, 1988). In this sense, closure may be seen as a density measure.

There are different views about cohesion, the most common is density and refers to the "number of ties among a set of nodes expressed as a function of the number of pairs of nodes" (Borgatti \& Li, 2009, pp. 11). Other concept of cohesion is the clique which refers to the maximal subset of nodes in which the density is $100 \%$. Usually, we don't find perfect cliques with real data; however there are some techniques to find imperfect cliques.

\section{A model of influence of the social network on psychological safety}

In this article we consider the social network as a mechanism by which information flows through communication. The communication networks are the mechanism that exposes the individuals to information, attitudinal messages and others' behaviours (Monge \& Contractor, 2001). It is also through communication (or the absence of it) that psychological safety reveals itself. For example a team with low psychological safety is likely to have less communication levels because its members assume passive communication behaviours in order to protect their personal image.

On the other hand, several researchers (e.g. Rice \& Aydin, 1991; Fulk, 1993; Scherer \& Cho, 2003) argue that communication networks allow individuals to develop meanings about their social context.

Based on this we propose a model (see Figure 1) illustrating the impact of social network dynamics on the psychological safety of the team. The key concept underlying this impact is social contagion. In general we refer to social contagion as the process by which the team members adopt attitudes or behaviours of others in the social network with which they communicate (Scherer \& Cho, 2003). In this sense we focus more on the process by which a 
team becomes psychologically safe than on the antecedents or outcomes of psychological safety.

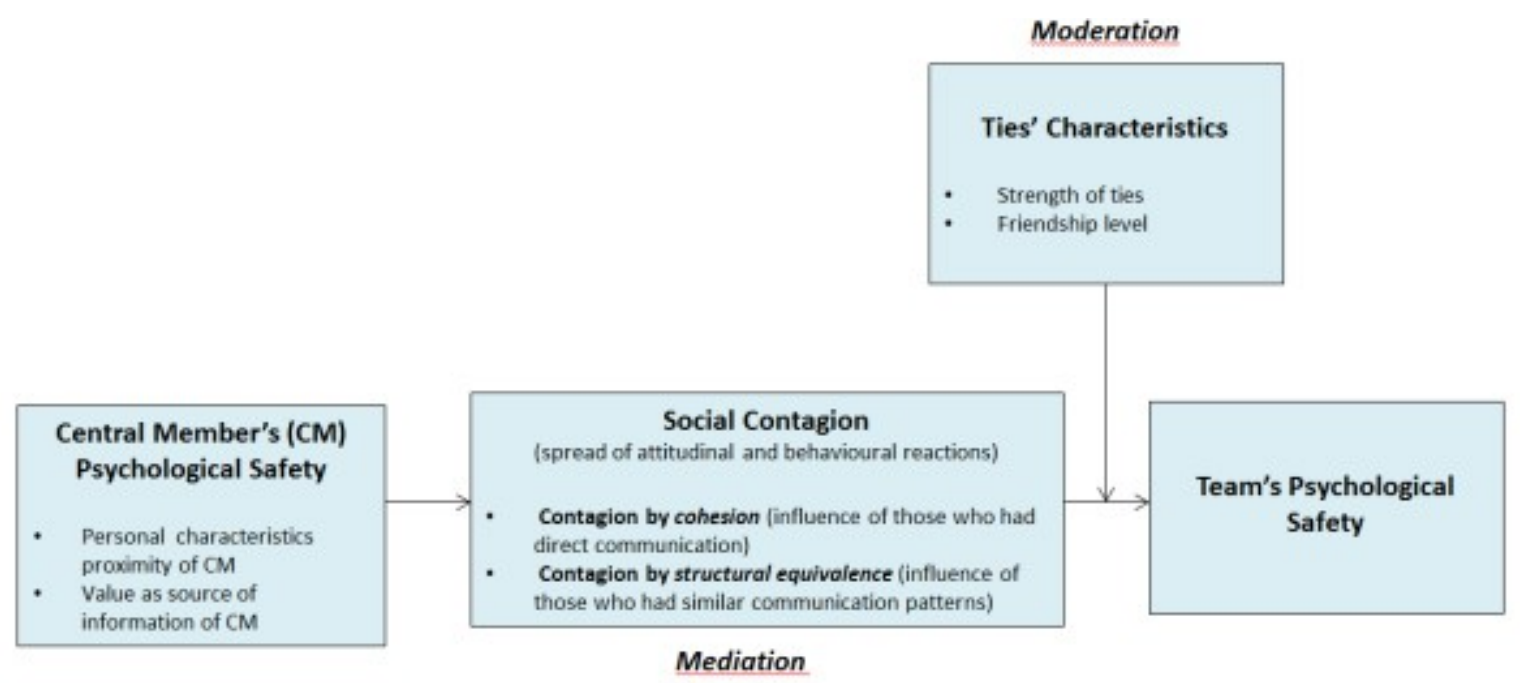

Figure 1. Model of the influence of social network on team psychological safety

This process is influenced by variables at three levels:

- the characteristics of the central member,

- the social contagion process and,

- the characteristics of ties.

As for the characteristics of central members we propose three variables. First, we expect that the psychological safety of the central member will impact on the psychological safety of the network through a social contagion process. This happens because, according to Freeman (1978), the central member will be the one that is closest to the other members of the network. As such, the central member assumes a position that allows him/her to interact with a large number of team members in a shortest path. On the other hand, the mere exposition to others may be not enough to the contagion to occur. From this perspective, we sustain that two characteristics of the central member mediate the impact of the node's psychological safety on the network psychological safety - the value of the central member as a source of information and the strength of the proximity to the node's personal characteristics.

In this sense, and according to Rice and Aydin (1991), the mere exposition to others is not enough for social information to have an effect. Individuals must, in some way, value others as a source. This means that the target of influence must see the source of information as trustworthy and valuable. For example, a civil construction worker talking with a civil engineer about construction tends to see him as a valuable source of information because he is an expert. On the other hand, the strength of the proximity to the personal characteristics of a 
central member is determined by the similarities between the characteristics of that central member and those of others within the network, evaluated by a comparison process.

The contagion process occurs by two processes: contagion by cohesion and contagion by structural equivalence (Burkhardt, 1994). The contagion by cohesion refers to the influence of those who had direct communication and the contagion by structural equivalence refers to the influence of those who had similar communication patterns.

At the level of tie properties we propose two variables that mediate the influence of the psychological safety of the central member and the psychological safety of his networkstrength of ties and friendship levels. We refer strength of ties as the frequency of interaction between nodes. As previously mentioned, network density refers to "the number of ties among a set of nodes expressed as a function of the number of pairs of nodes" (Borgatti \& Li, 2009, pp. 11). The friendship levels refer to the perception of friendship that an individual develops with whom he interacts. We now proceed to describe in more detail the influence of social contagion on the team's psychological safety and the influence of each group of variables on the social contagion process.

\subsection{The influence of the central member}

Traditionally, the influence of an individual on the attitudes, beliefs and behaviours of the group has been studied by leadership scholars. For leadership theorists, the leader is mainly the middle or top management who has the responsibilities to manage other people. Over the past 60 years leadership research has focused on the leader's characteristics or behaviours and contextual factors, ignoring the structure of interpersonal relationships. According to Brass and Krackardt (1999), the twenty-first century leader has to identify and nurture potential relationships, putting the right people together in the right place at the right time. Therefore, the structure of interpersonal relationships influences the leadership outcomes. On the other hand, centrality is the key component for leadership in organizations (Brass \& Krackardt, 1999) to the extent that there is a positive relationship between central network position and power and influence (e.g. Brass, 1992). Thus the informal position of individuals (the social structure) is more important than the hierarchical position (formal structure).

In this sense the position within the network will be important for individuals to be considered as leaders by others. We therefore assume that any member of the team may occupy a central position within the network regardless of his formal position, enabling his influence on other's attitudes, beliefs and behaviours.

On the other hand, and according to the concept of closeness suggested by Freeman (1978), the central member is the one with shorter distances to or from all other nodes. Thus, the central member will communicate with a larger number of actors than others. However, 
because he is the one with the shortest path to all the others, the information that he provides will be less exposed to the interference generated by the interactions with several members. As such, the central member will be in a privileged position to influence the entire network. So taking into account the social contagion process and the privileged position of the central nember in terms of communication, we may advance the following proposition:

- P1: The psychological safety of the team is positively related with the psychological safety of the central member.

However, the influence of the central member may be facilitated by his/her characteristics and by context. Considering the social information processing theory, already described in this paper, the influence of a person in others beliefs, attitudes and behaviours can be facilitated in two different ways. First, individuals must be proximate to the attitudes, information, or behaviour of others to be exposed to social information (Salancik \& Pfeffer, 1978). According to Rice and Aydin (1991, pp. 220), proximity is defined as the "extent to which one could be exposed to social information in a given social system." Other authors (e.g. Borgatti \& Foster, 2003; Ibarra, 1992) refer to another kind of proximity which results from the homophily principle, i.e. the tendency for people to interact more with those of their own kind (e.g. individual characteristics as race, gender, educational class, organizational unit). In this sense, we may consider two kinds of proximity:

- opportunity proximity, related with the opportunity that individuals have to interact and

- personal characteristics proximity, related to the individual similarities between them.

In addition, the individual must value others as a source in some way, for the social information to have an effect (Rice \& Aydin, 1991). We must not confuse the "value" of the source with the strength of the closeness in terms of personal characteristics. The process by which an individual establishes the other's value depends merely on the evaluation of the other's qualities. In contrast, the strength of proximity of personal characteristics is determined by similarities between an individual and others evaluated by a comparison process. For example, a student may see the lecturer as a valuable source of information, not because of the similarities between them but because of his status. 
In a social network based on the communication between actors, the central member is the one that communicates with more others and that can reach them in the shortest path. In this sense we may consider that the central member is the one seen as a valuable source of information and/or is the one that is the closest (opportunity or personal characteristics proximity) to a higher number of network members. In this sense, based on social networks, we identify three components that are important to an actor to become central in communication:

- opportunity to contact with a large number of members;

- similarity to a larger number of actors and

- the actor is seen by others as a valuable source of information.

This leads us to the following propositions:

- P2a: The opportunities for the central member to contact with others have a positive impact on the strength of ties.

- P2b: The proximity to the personal characteristics of the central member has a positive impact on the psychological safety of the team.

- P2c: The value of the central member as a source of information moderates has a positive impact on the psychological safety of the team.

\subsection{The influence of social contagion}

In the literature, different terms are used to describe the process of influence of an actor by another. The contagion approach seeks to explain the knowledge, attitudes and behaviour of the organizational members on the basis of information, attitudes, and behaviour of others within the network to which they are linked (Monge \& Contractor, 2001). On the other hand, social contagion suggests that actors adopt the attitudes or behaviours of the others in the social network with whom they communicate (Scherer \& Cho, 2003). This influence may be conscious or unconscious and does not require that there is any intention to influence. Lenders (2002) also advocate that it does not matter whether the influence is intentional or unintentional. Therefore, the communication is the most important aspect for the contagion to occur regardless of intentionality.

Contagion can be distinguished into contagion by cohesion and contagion by structural equivalence (Burkhardt, 1994). The contagion by structural equivalence refers to the influence 
of those who had similar communication patterns. On the other hand, contagion by cohesion refers to the influence of those who had direct communication.

In this sense, the network has a special role since it is the mechanism that exposes individuals to information, attitudes, behaviours and beliefs through the contact with others. Thus, social network analysis allows us to identify the sources of information of each team member as the mere exposure of others' attitudes, behaviours and beliefs will influence one's own attitudes, behaviours and beliefs.

Other framework referring to the contagion is the social information processing approach of Salancik and Pfeffer (1978). This approach proceeds from the fundamental premise that individuals, as adaptive organisms, adapt attitudes, behaviour and beliefs to their social context and to the reality of their own past and present behaviour and situation. Therefore it emphasises the importance of social context as a determinant of an individuals' attitudes, behaviours and beliefs. In this sense, to understand individual behaviour we must understand the informational and social environment within which that behaviour occurs and to which it adapts. According to this approach communication is fundamental for individuals to gather information about social context.

Concerning the influence of social networks on social information processing, Rice and Aydin (1991) concluded that relational proximity (i.e. communication) is more important than spatial proximity, concerning the social information processing. Once again, communication between individuals is pointed out as fundamental to predict their attitudes, behaviours and beliefs. Indeed, Rice and Aydin (1991) support that the social networks based on communication are better predictors of social information processing than others, such as, for instance, those based on spatial positioning.

All these perspectives emphasize the influence of social and symbolic processes, like communication between individuals, on developing patterns of shared cognitions and behaviours. On the other hand, these theories focus more on different aspects of social construction process than on conflicting premises (Fulk, 1993). Finally, all these perspectives advocate that communication is essential for individuals to develop meanings about their social context.

Considering psychological safety as a shared belief about the social context of individuals, we thus expect that the pattern of the network of communication strongly influences psychological safety. On the other hand, according to social contagion theory (Scherer \& Cho, 2003), people tend to adopt the attitudes or behaviours of others in the social network with whom they communicate. So communication is the minimum condition to enable social contagion.

As such, we may expect that information shared by individuals may influence psychological safety, especially when it reports to situations of personal risk-taking with a negative impact. 
For example, a worker who faces a situation where he is attacked by colleagues because he pointed out a mistake tends to develop low psychological safety. If this worker reports this situation to another, the listener may develop a defensive behaviour (i.e. develop low psychological safety) in order to avoid the same negative reactions. In view of the above mentioned, we may advance the following proposition:

- P3: The social contagion process (contagion by cohesion and contagion by structural equivalence) mediates the central member's influence on the psychological safety of the team.

\subsection{The influence of tie characteristics}

According to Granovetter (1973, pp. 1361) "the strength of a tie is a (probably linear) combination of the amount of time, the emotional intensity, the intimacy (mutual confiding), and the reciprocal services which characterize the tie". On the other hand, Monge and Contractor (2001) refer to the strength of tie as different measures, like amount of time, emotional intensity, intimacy and frequency, among others. The main difference between these two authors is that the first assumes that tie strength results from the combination of the dependent measures. Contrarily, the second assumes that tie strength may be measured using different and independent measures. On the other hand, McPherson, Popielarz and Drobnic (1992) consider a multi-dimensional concept of strength of ties. These authors refer three aspects of tie strength:

- the frequency of contact between two nodes,

- the density of connection between the nodes, and

- the sociodemographic distance between the nodes.

The contact frequency refers to the frequency of interaction, i.e. number of times a node communicates with another. High frequency of interaction will increase the amount of shared information, the emotional bond and so forth (McPherson et al., 1992). In line with this, Scherer and Cho (2003) found evidences that to be effective, communication must be frequent. Individuals who communicate with each other frequently are more likely to share knowledge effectively than those who communicate infrequently (Reagans \& McEvlily, 2003). In this sense, the mere communication between individuals may not be enough for the contagion to occur if it is infrequent.

The density of connection between nodes refers to network density, i.e. the extent to which people are interconnected. According to McPherson et al. (1992), when the members are more interconnected they are likely to share tastes, outlooks, and other features, since their 
contacts also interact with each other. A denser network provides redundant information to the members, which may contribute to the emergence of a shared vision of the environment. On the other hand, a network with a higher number of "connections" will increase the psychological safety flow across the network. Finally, a high network density prevents the emergence of cohesive subgroups that may stay away from the influence of the central member, and may develop a shared understanding of the environment that is different from the rest of the network and/or from other cohesive subgroups. In this sense, network density will enable the effect of the central member. This means that if the central member does not feel psychologically safe but the network has a low density the contagion of the whole network will be lower.

The sociodemographic distance refers to the dissimilarity of members in terms of demographic characteristics, such as education and age. Higher levels of dissimilarity in some variables may lead to the emergence of cohesive subgroups because the individuals with similar characteristics (different from the remainder group) will group themselves in subgroups. For instance, members in their 20 s may prefer to interact to each other than with members in their 50s. However this is more evident concerning informal interactions. In terms of work related interactions, the members may have to interact with others from a different age in order to accomplish their tasks and goals. Thus homogeneous groups will be those with stronger ties.

There are many consequences of having a network with strong ties that may influence the psychological safety contagion process. For instance, at an ego level, McPherson et al. (1992) found evidence that strong ties with other members increase the duration of membership of team members. On the other hand, Lee and Kim (2010) evidenced that strong ties will promote the access to others in order to offer or receive social support. In turn, Granovetter (1973) states that if the members of a social group share strong ties between each other, then they tend to possess similar norms, attitudes, behaviours, and knowledge. Hence, the mere communication between individuals may not be enough for the contagion to occur. The contact frequency must be high, the network must be dense and the group must tend to be homogeneous in terms of sociodemographic characteristics. In sum, the strength of ties has to be strong. This leads us to the following proposition:

- P4: The strength of ties moderates the social contagion process impacting the team's psychological safety

Another characteristic of ties that may influence the contagion process is the level of friendship. A study led by Schulte, Cohen and Klein (2010), in which they advance with three sociopsychological mechanisms describing the influence of network ties on psychological safety. 
First, the number of friendship ties to teammates will influence the perception of psychological safety, i.e. a greater number of friendship ties will be related with greater psychological safety feelings (retrospective sense-making). Second, also if a person has extended ties of friendship from many of his or her teammates, he or she will likely to infer that the team is psychologically safe (reaction). This happens because this person will tend to receive a lot of expressions of friendship from teammates. Finally, Schulte et al. (2010) advocate that the psychological safety of a person will be positively related with the psychological safety of those to whom he/she sends friendship ties. In this sense, the friendship network contributes to psychological safety.

In line with this perspective May et al. (2004) showed a positive relation between employees' perceptions of how rewarding their co-worker relations were and their perceptions of psychological safety. Trust may contribute to psychological safety (Edmondson, 1999), so the trust inherent to a friendship relation may foster psychological safety. So, if a central member in a communication network is also a central member in a friendship network he or she will have a greater influence in the psychological safety of the network. However, friendship shouldn't be faced as a dichotomous dimension (to have or not have a friendship tie) but rather as a continuous variable. This means that people may feel different levels of friendship depending on the person with whom the friendship tie is established. Thus for the contagion process to occur the level of friendship feelings that an individual has towards others with whom he or she communicates is more important than whether there are or there are not friendship ties. So the interaction gives the indication of the communication and the friendship gives clues about the quality of the relationship. This leads us to the following proposition:

- P5: The friendship level moderates the social contagion process impacting the team's psychological safety.

\section{Discussion}

From our point of view, this integration of social network analysis with team psychological safety pushes the theory forward on two different fronts. First, it shifts the focus of the study of psychological safety from an input and output perspective to a process view. We propose a model based on the interactions between team members to illustrate the process by which a team becomes psychologically safe. Thus, our model proposes that central team members (central members) play a key role on the psychological safety of a team. On the other hand, network characteristics, namely friendship and the strength of ties moderate the social contagion process by which the central member contributes to the psychological safety of the whole team. Second, this paper focuses more on the process by which a team becomes psychologically safe than in the antecedents and consequences of psychological safety. Regarding this, the social contagion mediates the influence of central member's on the 
psychological safety of the team. This doesn't mean that it is not important to understand the antecedents and consequences of psychological safety; we just tried to fill a fundamental gap in the study of psychological safety, the influence that central member may have on team's psychological safety, the process by which that influence occur and moderator variables that contribute for that influence.

\subsection{Implications}

There are some potential implications from our propositions in terms of central member, network characteristics and context characteristics. Concerning the central member, future research might explore the role of the individual characteristics of the central member in the development of the psychological safety of the team. In this article we only include one personal characteristic of the central member - psychological safety. Indeed, all other characteristics, the personal characteristics of the node and the value of central member as a source of information are more related with the perceptions of others than with the node's "real" characteristics. So it would be important to explore the role of the other characteristics of the central member in the development of psychological safety. For instance, is the authenticity of central members related with higher levels of team psychological safety? It is also important to explore which behaviours of the central member most influence the psychological safety levels of the team. For example, it would be interesting to understand if the supportive behaviour of the central member influences the psychological safety of the team. Finally, it may also be important to understand if the formal position of the central member (e.g. formal leadership) on the organizational structure influences the contagion process.

In the present work, we advance with the strength of ties and network density as network characteristics that mediate the influence of the central member on the psychological safety of the team. However, it is important, in future research, to explore other network features or team characteristics that may directly or indirectly influence the contagion process. For instance, the number of nodes may influence network density as it is easier for members in small teams to interact with all other peers than for members in big teams. On the other hand, there are other variables in social network theory that may influence psychological safety. For example, we may ask if the number of bi-directional interactions will influence the contagion process.

In the model proposed here interaction opportunities are referred to as context characteristics that influence the strength of ties. Further research is needed to understand the variables that will enable these opportunities. For example, future research might explore the influence of the office layout on providing interaction opportunities. On the other hand, these interaction opportunities may be influenced by other contextual or situational aspects not related with space characteristics. 


\subsection{Concluding remarks}

In conclusion, an understanding of the influence of the social network on the team psychological safety give us important insights into the role of team member interactions in psychological safety. We considered that some team members (the most central) will play a key role in the construction of the psychological safety of a team. This understanding may lead us to a new way of approaching team management. Team managers can improve team performance by using social network analysis to identify central members, accessing their psychological safety, and improving the psychological safety of central members. They may also, for example, create interaction opportunities for team members to contact to each other. Finally, we hope that this work may contribute to the development of new intervention tools to improve team performance, enable organizational learning and team learning behaviours such as available time to interact.

\section{Acknowledgments}

The authors thanks to Fátima Paiva for the proofreading and the useful suggestions.

\section{References}

Baer, M., \& Frese, M. (2003). Innovation is not enough: Climates for initiative and psychological safety, process innovations, and firm performance. Journal of Organizational Behavior, 24(1), 45-68. http://dx.doi.org/10.1002/job.179

Borgatti, S.P., \& Li, X. (2009). On social network analysis in a supply chain context. Journal of Supply Chain Management, 45(2), 1-17.

Borgatti, S., \& Foster, P. (2003). The network paradigm in organizational research: A review and typology. Journal of Management, 29(6), 991-1013. http://dx.doi.org/10.1016/S01492063(03)00087-4

Brass, D. (1992). Power in organizations: A social network perspective. In Moore, G. \& Whitt, J. (Eds), Research in politics and society (pp. 295-323). Greenwich, CT: JAI Press.

Brass, D., \& Krackardt, D. (1999). The Social Capital of Twenty First Century Leaders. In J.G. Hunt, R.L. Philips \& L. Wong (Eds.), Out-of-the box leadership challenges for the 21st century army (pp. 179-194). Wagon Lane: Emerald.

Burkhardt, M.E. (1994). Social interaction effects following a technological change: A longitudinal investigation. Academy of Management Journal, 37: 868-896.

Burt, R. (2005). Brokerage and Closure: An Introduction to Social Capital. New York: Oxford University Press. 
Carmeli, A. (2007). Social capital, psychological safety and learning behaviours from failure in organisations. Long Range Planning, 40(1), 30-44.

Carmeli, A., \& Gittell, J. (2009). High-quality relationships, psychological safety, and learning from failures in work organizations. Journal of Organizational Behavior, 30(6), 709-729. http//dx.doi.org/ 10.1002/job565

Coleman, J. (1988). Social capital in the creation of human capital. The American Journal of Sociology, 94, S95-S120.

Edmondson, A. (2003). Managing the risk of learning: Psychological safety in work teams. In M. West, D. Tjosvold \& K. Smith (Eds.). International Handbook of Organizational Teamwork and Cooperative Work (pp. 255-275). London: Wiley.

Edmondson, A. (1999). Psychological safety and learning behaviour in work teams. Administrative Science Quarterly, 44(2), 350-383.

Freeman, L. (1978). Centrality in social networks conceptual clarification. Social Networks, $1(3), 215-239$.

Fulk, J. (1993). Social construction of communication technology. Academy of Management Journal, 36(5), 921-950.

Gist, M.E., Locke, E.A., \& Taylor, M. (1987). Organizational behavior: Group structure, process and effectiveness. Journal of Management, 13, 237-257.

http://dx.doi.org/10.1177/014920638701300204

Granovetter, M. (1973). The strength of weak ties. American Journal of Sociology, 78(6), 1360-1380.

Ibarra, H. (1992). Homophily and differential returns: Sex differences in network structure and access in an advertising firm. Administrative Science Quarterly, 37, 422-447.

Kozlowski, S.W.J., \& Ilgen, D.R. (2006). Enhancing the effectiveness of work groups and teams. Psychological Science in the Public Interest, 7, 77-124.

Losada, M., \& Heaphy, E. (2004). The role of positivity and connectivity in the performance of business teams. American Behavioral Scientist, 47(6), 740-765.

http://dx.doi.org/10.1177/0002764203260208

Lee, J., \& Kim, S. (2010). Exploring the role of social networks in affective organizational commitment: network centrality, strength of ties, and structural holes. The American Review of Public Administration, 41(2), 205-223. http://dx.doi.org/10.1177/0275074010373803

Lusher, D., Robins, G., \& Kremer, P. (2010). The application of social network analysis to team sports. Measurement in Physical Education and Exercise Science, 14(4), 211-224. http://dx.doi.org/10.1016/S0378-8733(01)00049-1 10.1080/1091367X.2010.495559 
May, D., Gilson, R., \& Harter, L. (2004). The psychological conditions of meaningfulness, safety and availability and the engagement of the human spirit at work. Journal of Occupational and Organizational Psychology, 77(1), 11-37. http://dx.doi.org/10.1016/S0378-8733(01)00049-1 10.1080/1091367X.2010.49555910.1348/096317904322915892

McPherson, J. M., Popielarz, M., \& Drobnic, S. (1992). Social networks and organizational dynamics. American Sociological Review, 57, 153-170. http://dx.doi.org/10.1016/S03788733(01)00049-110.1080/1091367X.2010.49555910.1348/09631790432291589210.2307/2096202

Monge, P., \& Contractor, N. (2001). Emergence of communication networks. In F. Jablin \& L. Putnam (Eds.). Handbook of Organizational Communication (2nd ed.). Thousand Oaks, CA: Sage.

Probst, T., \& Estrada, A. (2010). Accident under-reporting among employees: Testing the moderating influence of psychological safety climate and supervisor enforcement of safety practices. Accident Analysis \& Prevention, 42(5), 1438-1444.

http://dx.doi.org/10.1016/j.aap.2009.06.027

Reagans, R., \& McEvily, B. (2003). Network structure and knowledge transfer: The effects of cohesion and range. Administrative Science Quarterly, 48, 240-267.

Rice, R., \& Aydin, C. (1991). Attitudes toward new organizational technology: network proximity as a mechanism for social information processing. Administrative Science Quarterly, 36, 219-244.

Robins, D. \& Kremer, P. (2010) The application of social network analysis to team sports. Measurement in Physical Education and Exercise Science, 14(4), 211-224.

Salancik, G., \& Pfeffer, J. (1978). A social information processing approach to job design and task design. Administrative Science Quarterly, 23, 224-253.

Scherer, C., \& Cho, H. (2003). A social network contagion theory of risk perception. Risk Analysis, 23(2), 261-267.

Schulte, M., Cohen, N., \& Klein, K. (2010). Coevolution of network ties and perceptions. Organization Science, 1-18.

Stacey, R. (1996). Strategic management and organisational dynamics (2nd ed.). London: Pitman.

Journal of Industrial Engineering and Management, 2014 (www.jiem.org)

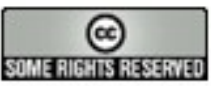

Article's contents are provided on a Attribution-Non Commercial 3.0 Creative commons license. Readers are allowed to copy, distribute and communicate article's contents, provided the author's and Journal of Industrial Engineering and Management's names are included. It must not be used for commercial purposes. To see the complete license contents, please visit 